\title{
Looking at Electron Dynamics to Better Understand Photo-Active Materials ${ }^{\dagger}$
}

\author{
Sayantan Bhattacharya ${ }^{1}$, Arthur Graf ${ }^{1}$, Chloe Britton-Powell ${ }^{1}$, Sami Rtimi ${ }^{2}$, Adrien Chauvet ${ }^{1, *}$ \\ 1 The University of Sheffield, Chemistry Department, Dainton Building, Sheffield S3 7HF, UK; \\ sayantan.bhattacharya@ sheffield.ac.uk (S.B.); agraf2@sheffield.ac.uk (A.G.); cbritton-powell1@ @heffield.ac.uk (C.B.P.) \\ 2 EPFL-STI-IMX-LTP, Station 12, Ecole Polytechnique Fedé rale de Lausanne, 1015 Lausanne, Switzerland; \\ sami.rtimi@epfl.ch (S.R.); \\ * Correspondence: a.chauvet@sheffield.ac.uk; \\ $\dagger$ Presented at Materials Chemistry and Physics (Materials Chemistry 2020) - International e-Conference
}

Received: 16.09.2020; Revised: 20.09.2020; Accepted: 24.09.2020; Published: 27.09.2020

\begin{abstract}
The generation of free and energetic electrons is the key to the next generation of photovoltaic and photo-catalysts. In order to produce such electrons, the sunlight is so far the most promising sustainable source of energy. However, the conversion efficiency from light to the liberation of a functional electron depends on numerous factors and processes that are difficult to account for. One of the reasons is the difficulty of monitoring the early electron dynamics such as the charge separation event and the subsequent electronic relaxation and migration. Here, I propose the use of ultrafast transient absorption spectroscopy to watch these electronic events "live" in the femto- to nano-seconds $\left(10^{-15}-10^{-9} \mathrm{sec}\right.$.) time scales. In this presentation, I will demonstrate this technique on iron and copper oxides thin films. In this study, we produce ultrashort laser pulses to mimic sunlight and trigger charge separations that ultimately lead to the generation of the reactive oxygen species that confer the films their antibacterial properties. With this technique, we are able to harvest information such as the charge separation efficiency, electron dynamics, excited state's bandgap energy, and even material deformation upon photo-excitation, all of which are key to understand, control and enhance the conversion process.
\end{abstract} Keywords: ultrafast spectroscopy; transient absorption; electron dynamics; thin film; photoactive material.

(C) 2020 by the authors. This article is an open-access article distributed under the terms and conditions of the Creative Commons Attribution (CC BY) license (https://creativecommons.org/licenses/by/4.0/).

\section{Funding}

This research was funded by the Engineering and Physical Sciences Research Council (EPSRC), grant number EP/R042802/1, and EP/R045305/1.

\section{Acknowledgments}

The authors thanks all the members of the Lord Porter Ultrafast Laser Spectroscopy Laboratory in Sheffield for their support and dedication.

\section{Conflicts of Interest}

The authors declare no conflict of interest. 